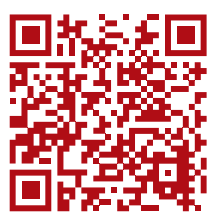

\title{
Fascitis necrosante por Klebsiella pneumoniae secundaria a inyección intramuscular glútea manejada con sistema de presión negativa
}

\author{
Necrotizing fasciitis due to Klebsiella pneumoniae secondary to intramuscular \\ gluteal injection managed with a negative pressure system \\ Dr. Julio Ángel Barajas-Puga,* Dr. Ricardo Barajas-Rico,* Dr. Claudio López-Giacoman ${ }^{\ddagger}$
}

\footnotetext{
Palabras clave: Fascitis necrosante, Klebsiella pneumoniae, inyección intramuscular, terapia de presión negativa.

Keywords: Necrotizing fasciitis, Klebsiella pneumoniae, intramuscular injection, negative pressure therapy.
}

\section{RESUMEN}

La fascitis necrosante es una infección grave, poco frecuente y de progresión rápida, cuyo diagnóstico es difícil en estadios tempranos. Produce trombosis de la microcirculación con afectación del tejido celular subcutáneo y la fascia muscular; se asocia con una elevada mortalidad. Los casos de fascitis necrosante documentados en los que la Klebsiella pneumoniae es el agente etiológico son extremadamente raros, con una tasa de mortalidad de $70 \%$. Presentamos el caso de una mujer de 57 años, quien desarrolló fascitis necrosante después de una inyección intramuscular en el glúteo derecho. Fue manejada con terapia de presión negativa en el Hospital General de Zacatecas «Luz González Cosío» con buena evolución, obteniendo resultados funcionales y estéticos satisfactorios. La terapia de presión negativa facilitó considerablemente el tratamiento y evolución de la paciente, favoreciendo la granulación de la herida y el cierre definitivo. Destacamos la utilidad de la terapia de presión negativa en el manejo y curación de heridas, evitando cirugías reconstructivas complejas.

\section{ABSTRACT}

Necrotizing fasciitis is a serious, rare and fast progressing infection, which that is difficult to diagnose in the early stages. It produces thrombosis of the microcirculation with involvement of the subcutaneous cellular tissue and muscle fascia and is related to high mortality. Documented cases of necrotizing fasciitis in which Klebsiella pneumoniae is the etiologic agent are extremely rare and have a mortality rate of $70 \%$. We present the case of a 57-year-old woman who developed necrotizing fasciitis after an intramuscular injection in the right gluteus. She was handled with negative pressure therapy at the General Hospital of Zacatecas «Luz González Cosío» showing a good progress, with satisfactory functional and aesthetic results. The negative pressure therapy facilitated the treatment and evolution of the patient considerably, favoring the granulation of the wound and the definitive closure. We underline highlight the usefulness of negative pressure therapy in the management and healing of wounds, staving off avoiding complex reconstructive surgeries.

\section{INTRODUCCIÓN}

* Residente de cirugía general, Departamento de Cirugía General. ₹ Médico adscrito al Departamento de Cirugía Plástica y Reconstructiva.

Hospital General de Zacatecas «Luz González Cosio». México.

Recibido: 11 marzo 2021 Aceptado: 17 mayo 2021
$\mathrm{L}$ a fascitis necrosante (FN) está considerada ـ como una urgencia médica por tratarse de una infección grave de los tejidos blandos que afecta la fascia y el tejido celular subcutáneo, y ocasiona necrosis de los mismos. Se asocia a una alta mortalidad por el compromiso sisté- mico y rápida progresión, con una incidencia anual de 0.4 por cada 100,000 habitantes. Los pacientes inmunocomprometidos son un grupo de riesgo. La FN se divide en cuatro subtipos: tipo I polimicrobiana, tipo II estreptocócica, tipo III por gramnegativos y tipo IV fúngica. ${ }^{1}$ El proceso fisiopatogénico inicia en la fascia superficial, donde ocurre proliferación bacteriana

Citar como: Barajas-Puga JÁ, Barajas-Rico R, López-Giacoman C. Fascitis necrosante por Klebsiella pneumoniae secundaria a inyección intramuscular glútea manejada con sistema de presión negativa. Cir Plast. 2021; 31 (2): 68-71. https://dx.doi.org/10.35366/102748 
y se generan toxinas que permiten la extensión de la enfermedad. La proliferación produce necrosis de la fascia superficial y trombosis en la microcirculación, lo que ocasiona isquemia del tejido celular subcutáneo, progresa en forma vertical y afecta planos profundos y dermis superficial, por lo que aparece la necrosis cutánea y ulceración. ${ }^{2}$ La presentación clínica característica consiste en el edema indurado que excede el área de eritema, calor y aparición de flictenas y crepitación subcutánea. La sintomatología muestra dolor desproporcionado y el diagnóstico se debe hacer a partir de una alta sospecha clínica y confirmación por intervención quirúrgica. Si la sospecha es alta son de gran ayuda métodos de imagen como la resonancia magnética (RM) y la tomografía computarizada (TC) y su realización no debe retrasar la intervención quirúrgica. ${ }^{3,4} \mathrm{La} \mathrm{FN}$ por Klebsiella pneumoniae es extremadamente rara, con un desarrollo rápido que ocurre en pacientes inmunodeprimidos. Se presenta como una enfermedad letal, con una tasa de mortalidad de $70 \%$. Una vez establecida, progresa rápidamente con un curso clínico fulminante. ${ }^{5}$ El tratamiento se fundamenta en medidas de soporte vital, manejo hídrico, antibioticoterapia de amplio espectro con cobertura de aerobios Gram positivos, Gram negativos y anaerobios. La acción fundamental sigue siendo el precoz y amplio desbridamiento quirúrgico del tejido desvitalizado. ${ }^{6,7}$ La terapia de presión negativa (TDPN) consiste en la aplicación de un sistema de presión subatmosférica con esponja bacteriostática sobre la herida, con un sellado adhesivo estéril. Con esto, la circulación en la zona se incrementa, produciendo un aumento en la formación de tejido de granulación, disminución de la carga bacteriana, elimina el exceso de exudado de la herida y reduce el edema. ${ }^{8}$ El objetivo de este trabajo consiste en describir un caso infrecuente de FN y el uso de TDPN en el manejo de este tipo de heridas.

\section{CASO CLÍNICO}

Mujer de 57 años, con antecedentes de hipertensión y diabetes, quien refirió inyección intramuscular de una ampolleta de complejo B y dexametasona en el glúteo derecho 20 días previos a su admisión, después de la cual inició con dolor y extensión a la región lumbar derecha, fiebre no cuantificada que no cedía al uso de acetaminofén, así como imposibilidad para la deambulación, por lo que acudió al servicio de urgencias del Hospital General de Zacatecas «Luz González Cosío». A la exploración física se encontró neurológicamente desorientada, con mucosas deshidratadas, palidez generalizada de piel y tegumentos, con cardiopulmonar sin compromiso, abdomen asignológico. La región del glúteo derecho dolorosa a la palpación superficial, con coloración eritematosa, aumento de volumen y extensión hasta la región lumbar derecha $(20 \times 22 \mathrm{~cm})$, con fluctuación franca; el resto normal. Signos vitales: frecuencia cardiaca 120 latidos por minuto, frecuencia respiratoria 19 por minuto, temperatura $38^{\circ} \mathrm{C}$, tensión arterial 133/87 mmHg. Exámenes de laboratorio: hemoglobina $9.6 \mathrm{~g} / \mathrm{dL}$, hematocrito $30.2 \%$, volumen globular medio $86.5 \mathrm{fL}$, hemoglobina corpuscular media $27.5 \mathrm{pg}$, plaquetas $504 \times 10^{9} / \mathrm{L}$, leucocitos 19,100 células $/ \mathrm{mm}^{3}$, neutrófilos $86 \%$, linfocitos $8.6 \%$. Se realizó TC observando aumento de tamaño de la zona glútea con pérdida de las interfaces, así como colección glútea de 18 × 32 cm con extensión hasta la región lumbar del lado derecho (Figura 1), con datos sugestivos de FN (Figura 2).

Se realizó fasciotomía y necrosectomía extensas, drenaje de absceso glúteo y de región lumbar derecha, lavado quirúrgico y desbridamiento de tejidos blandos profundos. Se encontraron $300 \mathrm{~mL}$ de líquido purulento de franco, se colocó un sistema de TDPN utilizan-

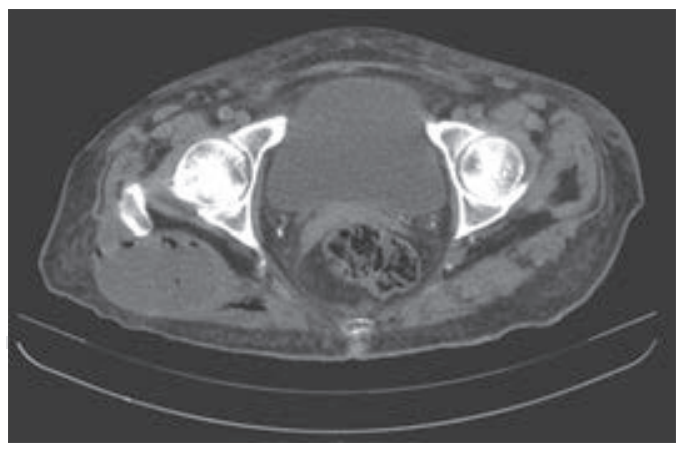

Figura 1: TC corte coronal. Se observa enfisema subcutáneo en tejidos blandos en glúteo derecho con engrosamiento de fascia superficial y profunda y colecciones líquidas por debajo de la fascia. 


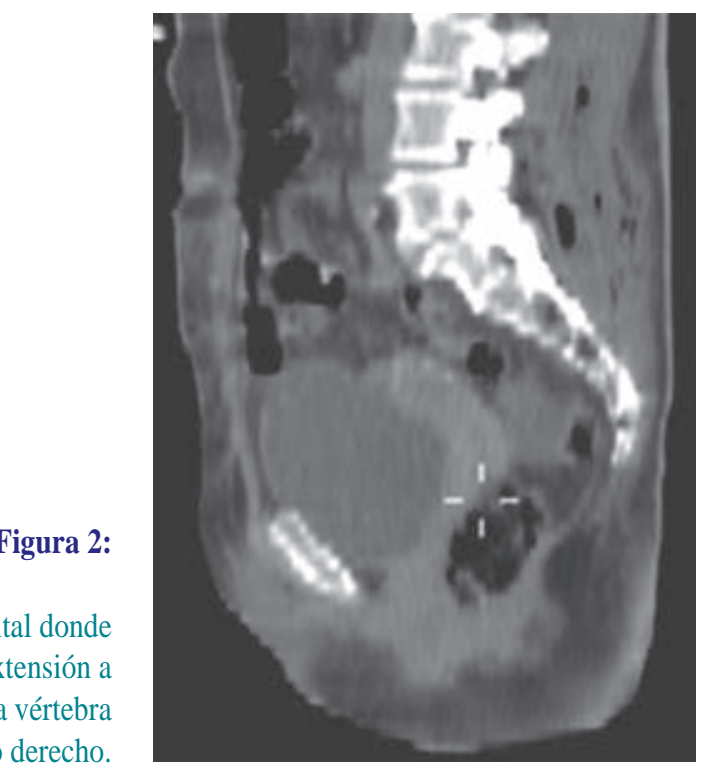

TC corte sagital donde se evidencia extensión a nivel de la cuarta vértebra lumbar del lado derecho.

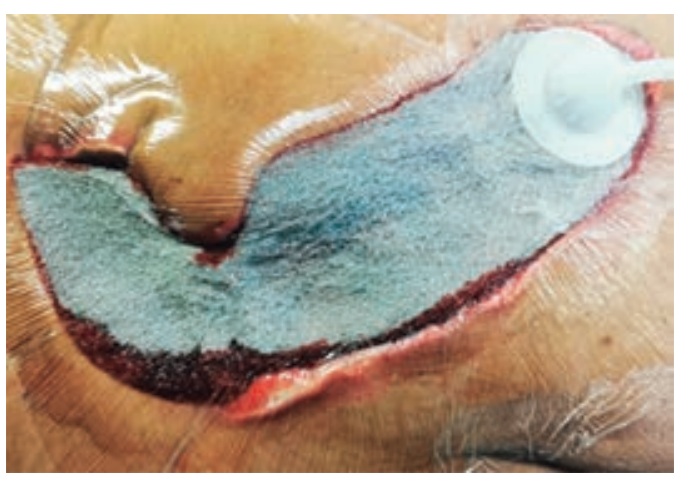

Figura 3: Colocación del sistema de presión negativa.

do esponja de plata (Figura 3), con confirmación microbiológica positiva a las 36 horas para Klebsiella pneumoniae sensible a ciprofloxacino. Se realizó desbridamiento exhaustivo y cambio de sistema de TDPN en tres ocasiones (cada 72 horas), con formación de tejido de granulación (Figura 4), para finalmente realizar cobertura definitiva en las áreas afectadas (Figura 5). La paciente evolucionó de manera satisfactoria y egresó a los 18 días después del cierre completo de la herida.

\section{DISCUSIÓN}

Presentamos un caso de FN manejado en el Servicio de Cirugía Plástica y Reconstructiva de nuestra unidad con base en antibioticoterapia, desbridamiento oportuno y el uso de TDPN que facilitó una evolución exitosa. La incidencia de la FN ha aumentado en los países occidentales en relación con el envejecimiento poblacional y enfermedades crónicas, lo cual está vinculado con el caso clínico. ${ }^{8,9}$ Mok MY y colaboradores asociaron el desbridamiento oportuno del tejido necrótico a menor mortalidad. ${ }^{10}$ Las inyecciones intramusculares pueden causar un traumatismo tisular severo, incluso con la adecuada aplicación puede ser un sitio de entrada local para una infección. ${ }^{11}$ La FN es frecuente en Asia y poco común en Occidente, con una mortalidad elevada. Las especies de Klebsiella están asociadas a infecciones del tracto urinario, neumonía y absceso hepático y es infrecuente en nuestro entorno hospitalario. ${ }^{12-14}$ La presión subatmosférica producida por la TDPN induce microdeformaciones en los tejidos, aumenta la proliferación y división celular, produce factores de crecimiento y angiogénesis, disminuye la cantidad de líquido inflamatorio, enzimas proteolíticas, metaloproteasas, mediadores proinflamatorios y citoquinas, reduce el edema $y$, al mantener la herida aislada, se reduce la carga bacteriana. La TDPN fue fundamental para el éxito en el tratamiento, manejo de la infección, cicatrización y dolor de nuestro caso, además facilitó el cierre de la herida, con una estancia hospitalaria de 2.5 semanas, que corresponde a lo descrito en la literatura ${ }^{15}$ y evitó cirugías

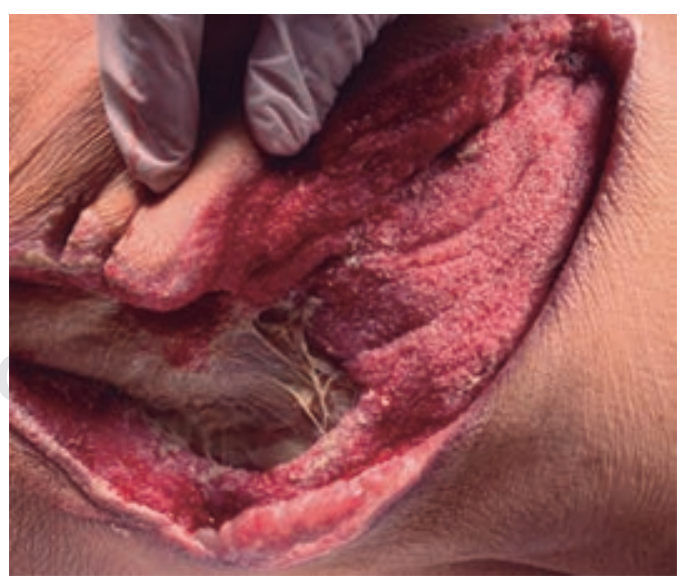

Figura 4: Formación de tejido de granulación en lecho quirúrgico. 


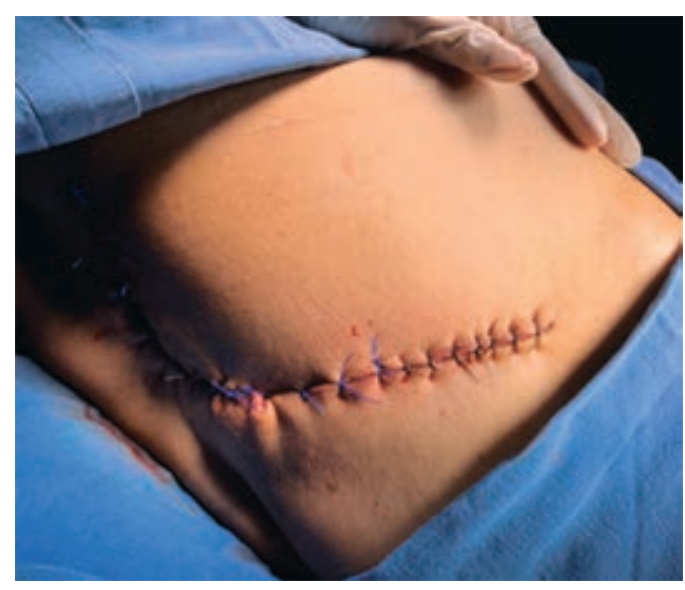

Figura 5: Cobertura definitiva de la herida quirúrgica.

reconstructivas complejas. Muchos autores han demostrado su eficacia en comparación con otros métodos. ${ }^{16,17}$

\section{CONCLUSIÓN}

La FN por Klebsiella pneumoniae es una infección grave de diagnóstico difícil, ocurre con factores predisponentes y subyacentes, con un curso rápido, agresivo y altas tasas de mortalidad. El diagnóstico y tratamiento médico-quirúrgico temprano son los factores más determinantes en la supervivencia. En el caso clínico expuesto, la TDPN fue fundamental en el tratamiento, evolución exitosa y cierre definitivo de la herida de la paciente, evitando cirugías reconstructivas complejas.

\section{REFERENCIAS}

1. Wong $\mathrm{CH}$, Khin LW, Heng KS, Tan KC, Low CO. The LRINEC (Laboratory Risk Indicator for Necrotizing Fasciitis) score: a tool for distinguishing necrotizing fasciitis from other soft tissue infections. Crit Care Med 2004; 32 (7): 1535-1541.

2. Parra Caballero P, Pérez Esteban S, Patiño Ruiz ME, Castañeda Sanz S, García Vadillob JA. Actualización en fascitis necrotizante. Semin Fund Esp Reumatol 2012; 13 (2): 41-88.

3. Chaudhry AA, Baker KS, Gould ES, Gupta R. Necrotizing fasciitis and its mimics: What radiologists need to know? Am J Roentgen 2015; 204(1): 128-139.

4. Stevens DL, Bryant AE. Necrotizing Soft-Tissue Infections. N Engl J Med 2017; 377 (23): 2253-2265.
5. Rahim GR, Gupta N, Maheshwari P, Singh MP. Monomicrobial Klebsiella pneumoniae necrotizing fasciitis: an emerging life-threatening entity. Clin Microbiol Infect 2018; 25 (3): 316-323.

6. Taviloglu K, Cabioglu N, Cagatay A, Yanar H, Ertekin C, Baspinar I et al. Idiopathic necrotizing fasciitis: risk factors and strategies for management. Am Surg 2005; 71 (4): 315-320.

7. Sarani B, Strong M, Pascual, Schwab CW. Necrotizing fasciitis: current concepts and review of the literature. J Am Coll Surg. 2009; 208 (2):279-288.

8. Al-Subhi FS, Zuker RM, Cole WG. Vacuum-assisted closure as a surgical assistant in life-threatening necrotizing fasciitis in children. Can J Plast Surg 2010; 18 (4): 139-142.

9. Roujeau JC. Critères cliniques et facteurs de risque Necrotizing fasciitis. Clinical criteria and risk factors. Ann Dermatol Venereol 2001; 128 (3 Pt 2): 376-381.

10. Mok MY, Wong SY, Chan TM, Tang WM, Wong WS, Lau CS. Necrotizing fasciitis in rheumatic diseases. Lupus 2006; 15(6): 380-383.

11. Holland C, Jaeger L, Smentkowski U, Weber B, Otto C. Septic and aseptic complications of corticosteroid injections: an assessment of 278 cases reviewed by expert commissions and mediation boards from 2005 to 2009. Dtsch Arztebl Int 2012; 109(24): 425-430.

12. Frick S, Cerny A. Necrotizing fasciitis due to Streptococcus pneumoniae after intramuscular injection of nonsteroidal anti-inflammatory drugs: report of 2 cases and review. Clin Infect Dis 2001; 33 (5): 740-744.

13. Pillans $\mathrm{PI}, \mathrm{O}^{\prime}$ Connor $\mathrm{N}$. Tissue necrosis and necrotizing fasciitis after intramuscular administration of diclofenac. Ann Pharmacother 1995; 29 (3): 264-266.

14. Persichino J, Tran R, Sutjita M, Kim D. Klebsiella pneumoniae necrotizing fasciitis in a Latin American male. J Med Microbiol 2012; 61 (Pt 11): 1614-1616.

15. Wong CH, Kurup A, Wang YS, Heng KS, Tan KC. Four cases of necrotizing fasciitis caused by Klebsiella species. Eur J Clin Microbiol Infect Dis 2004; 23 (5): 403-407.

16. Tenenhaus M, Rennekampff HO. Surgical advances in burn and reconstructive plastic surgery: new and emerging technologies. Clin Plast Surg 2012; 39 (4): 435-443.

17. Buendía Pérez J, Vila Sobral A, Gómez Ruiz R, Qiu Shao SS, Marré Medina D, Romeo M et al. Tratamiento de heridas complejas con terapia de presión negativa. Experiencia en los últimos 6 años en la Clínica Universitaria de Navarra, Pamplona (España). Cir Plast Iberolatinam 2011; 37(supl 1): 65-71.

Conflicto de intereses: Los autores declaran no tener conflicto de intereses.

Correspondencia:

Dr. Julio Ángel Barajas Puga

Prol. González Ortega Núm. 49,

Col. Centro Nochistlán

99900, Zacatecas, Zac. México.

E-mail: lafiestadelosgatos@gmail.com 